\title{
Les chamanes et nous
}

\section{Jean Martin}

Dr méd., membre de la rédaction

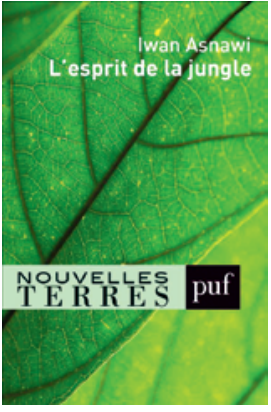

Iwan Asnawi L'esprit de la jungle

Paris: Presses universitaires de France/ Nouvelles Terres; 2019,126 pages
Traductrice de cet ouvrage, Sophie Swaton est philosophe et économiste à l'Université de Lausanne, spécialiste en durabilité. C'est en 2011 qu'elle rencontre Iwan Asnawi: ce thérapeute traditionnel indonésien vivant en Suisse depuis 1995 parvient à la soulager d'un status douloureux. Intéressée par son histoire, elle l'encourage à la mettre sur papier. Né en 1964 dans la jungle de Sumatra, Iwan Asnawi est marqué à vie par la nature et l'«esprit» de ce milieu qui l'a vu grandir. Après des études de droit, il est engagé dans l'opposition à la dictature brutale, durant trente ans, du général Suharto. Il épouse une Suissesse alémanique rencontrée dans le cadre d'Amnesty International. Iwan Asnawi retourne régulièrement dans son pays pour s'y ressourcer, notamment au contact de ses confrères dukuns (guérisseurs). Ses grands-parents étaient euxmêmes dukuns et une expérience forte en forêt, à 24 ans, l'a fait à son tour embrasser cette voie: «Si je devais définir un dukun, je dirais qu'il fait le pont entre le monde des esprits et le monde terrestre. Ce sont des personnes entièrement connectées à l'Univers et ressentant les liens entre les vivants», dit l'auteur. Il s'agit en fait de la définition universelle du chamane, qu'il/elle soit de Sibérie, d'autres régions nordiques ou des deux Amériques notamment.

\section{Si les cadres de référence et de pratique sont} totalement différents, les dukuns se fondent sur une déontologie semblable à la nôtre.

Dans ce livre autobiographique, Iwan Asnawi détaille son rapport étroit, presque intime, à la nature: "Dans nos croyances issues du syncrétisme, nous pensons que c'est au contact prolongé de la nature que celle-ci nous parle et nous révèle son savoir [...] La spiritualité n'est pas l'apanage des êtres humains. Chaque animal a un esprit, chaque arbre a une âme. On peut communiquer avec eux, de manière supra-sensorielle [...] Le pire c'est qu'à présent les gens croient que la nature est extérieure à eux. Ils oublient tout de leurs racines, de leurs savoirs intérieurs.» Si les cadres de référence et de pratique sont totalement différents, les dukuns se fondent sur une déonto- logie semblable à la nôtre: «Tu aides les gens. Mais tu dois promettre de ne pas les manipuler, de ne pas placer ton désir au-delà des leurs»; «Il n'est pas facile de soigner les siens [sa famille], l'implication émotionnelle étant souvent trop forte»; «La règle numéro un du don de guérisseur est la non-interférence avec le développement en cours de la personne traitée». S'agissant de l'information du patient, certains principes diffèrent partiellement de ce qui est reconnu aujourd'hui en Occident comme droits des malades:

"Cela a été un défi d'écouter sans objecter, d'apprendre une autre forme de savoir, de taire - partiellement - mes doutes.»

«Mon grand-père m'assistait et me rappelait que l'on ne peut pas tout dire. Désormais jamais je ne donne plus d'informations que ce que la personne souhaite recevoir.»

L'esprit de la jungle constitue une source intéressante aussi quant à l'histoire indonésienne récente, depuis l'indépendance en 1945 puis la période dictatoriale 1965-1998, à ses clans, à ses croyances et à sa nature menacée par les monocultures (huile de palme). On comprend que détruire la jungle, c'était détruire une spiritualité ancestrale. Mais l'auteur est animé par l'espoir d'un avenir possible: «Compte tenu de l'état de la planète, ose-t-on dire encore que les syncrétistes sont stupides?» Avenir qui dépend de la volonté et de la participation de chacune et chacun d'entre nous, conclut-il en dernière page: «Ce n'est qu'en prenant soin les uns des autres que nous parviendrons peut-être à relever le défi immense que doit affronter l'humanité.»

Dans la préface, Sophie Swaton explique l'enjeu de traduire un tel récit: «Cela a été un terrible défi pour moi d'écouter sans objecter, d'apprendre une autre forme de savoir, de taire - partiellement - mes doutes, de tenter de restituer l'essence de sa pensée [...] C'est un livre témoignage, un récit nécessairement elliptique.» Un livre décentrant et dépaysant, doté d'une vraie sagesse, même s'il est, pour nous Occidentaux, parfois très difficile de tout saisir et "prendre avec soi». 